\title{
¿Es posible prevenir la enfermedad mental grave?
}

\author{
Felipe Vázquez Estupiñán ${ }^{1}$
}

\section{LOS TRASTORNOS MENTALES GRAVES AFECTAN ENORMEMENTE A NIÑOS Y JÓVENES}

Los trastornos mentales graves son las enfermedades crónicas de los niños y jóvenes, de la misma forma como lo son el cáncer y las enfermedades cardiovasculares en los adultos. Los trastornos del espectro autista, las psicosis afectivas y no afectivas afectan aproximadamente al 2.5\% de la población adulta. ${ }^{1}$ El $75 \%$ de la carga de enfermedad global por trastornos mentales se genera en países en vías de desarrollo. Los trastornos neuropsiquiátricos son responsables de aproximadamente $20 \%$ de la carga de enfermedad en México; sin embargo, sólo se destina el $2 \%$ del presupuesto general de salud a estos problemas. ${ }^{2,3}$ Los trastornos mentales graves fracturan las carreras vocacionales y laborales de la gente joven en la etapa más productiva de sus vidas.

\section{LA IMPERIOSA NECESIDAD DE UN ENFOQUE PREVENTIVO}

La medicina, como práctica profesional al servicio de la sociedad, en el presente milenio busca el ideal de prevenir la discapacidad y el sufrimiento al reducir los factores de riesgo de las enfermedades graves en forma sistemática y oportuna, cubriendo a la totalidad de la población susceptible. Se ha logrado reducir la mortalidad y discapacidad asociada con las enfermedades cardiovasculares y el cáncer a partir del control de la presión arterial, uso de estatinas, reducción de la obesidad, práctica de actividad física, disminución del consumo de alcohol y tabaco, así como la detección oportuna de cáncer mamario, prostático y

\footnotetext{
${ }^{1}$ Maestro en Ciencias Médicas, Psiquiatra del Hospital Ángeles Clínica Londres.
}

Correspondencia:

Dr. Felipe Vázquez Estupiñán

Correo electrónico: felipevaz.doctor@gmail.com

Aceptado: 26-01-2017.

Este artículo puede ser consultado en versión completa en http:// www.medigraphic.com/actamedica cervicouterino en personas en riesgo; vacunación contra el virus del papiloma humano (VPH), educación en autoexploración y en prevención de enfermedades de transmisión sexual (ETS), entre otras medidas.

Siguiendo este modelo preventivo, en la psiquiatría contemporánea se busca identificar marcadores biológicos y datos clínicos que permitan establecer etapas de los principales trastornos con el fin de plantear acciones adecuadas para cada una. ${ }^{4}$ En este sentido, cuando un joven de 18 años ingresa a un hospital psiquiátrico con un cuadro agudo de psicosis (ideas delirantes, alucinaciones, inquietud motora, insomnio), el sistema preventivo no ha sido efectivo. ¿Es acaso posible prevenir las psicosis?

\section{SÍ ES POSIBLE. ¿QUÉ TIPOS DE PREVENCIÓN EXISTEN?}

La psiquiatría preventiva señala tres tipos de prevención: la universal dirigida a toda la población, la dirigida a la población en riesgo y la indicada en la población enferma. Aplicando este modelo a la esquizofrenia y el trastorno bipolar — dos trastornos graves asociados a discapacidad, internamientos y conducta suicida- podemos comentar los siguientes antecedentes:

\section{PREVENCIÓN UNIVERSAL}

La alfabetización en salud mental para reconocer los signos y síntomas tempranos del trastorno mental y dónde buscar ayuda es indispensable para la sociedad en su conjunto. La vigilancia médica prenatal, una adecuada alimentación durante el embarazo y una buena atención obstétrica serían algunas líneas de defensa para la prevención de la enfermedad mental. La desnutrición intrauterina se ha asociado a problemas metabólicos, como obesidad y diabetes, pero también con depresión y esquizofrenia en la edad adulta. ${ }^{5,6}$ El estudio de Nicodemus ${ }^{7}$ demostró un vínculo entre los genes AKT1, BDNF, DTNBP1 Y GRM3, involucrados en la función neurovascular o regulados por la hipoxia, interactuando con las complicaciones obstétricas graves para incrementar el riesgo de la esquizofrenia.

Medina-Mora ${ }^{8}$ y otros investigadores demostraron recientemente la necesidad urgente de prevenir el abuso infantil como imperativo para reducir el riesgo de psicosis. 


\section{PREVENCIÓN DIRIGIDA}

Se hace mucha investigación de variantes genéticas asociadas a trastornos mentales; pero, lamentablemente, no se hace la suficiente; en particular, si reconocemos que la variabilidad genética entre las poblaciones (europeas, asiáticas, latinoamericanas) dificulta la interpretación y aplicación de los hallazgos a la clínica. ${ }^{4}$ Los descendientes de las familias en las que existen miembros afectados con trastorno mental grave tienen un mayor riesgo de padecer estos problemas, por lo que se consideran en una primera etapa, en la que es posible intervenir a través de medidas nutracéuticas ${ }^{9}$ y cognitivas, así como participar en protocolos de estimaciones combinadas de riesgo genético y clínico, protocolos de detección temprana que identifiquen actividad glial incrementada, mediciones volumétricas de la corteza y estudios neurofisiológicos, entre otros.

Le Niculescu, ${ }^{10}$ abrevando en el trabajo seminal de Caspi ${ }^{11}$ en las relaciones genes-medio ambiente para explicar los trastornos mentales, propone un modelo predictivo de la conducta suicida que incluye transiciones de ausencia de ideación suicida a presencia (CADM1, CLIP4, DTNA, KIF2C), y de ideación a intento o suicidio consumado (IL6, MBP). Las vías biológicas incluyen la respuesta inmune e inflamatoria, la señalización mediada por mTOR, la regulación de factores de crecimiento, MAOB, genes de la apoptosis y genes asociados a la regulación del $\mathrm{pH}$ extracelular cerebral (SLC4A4). Los adolescentes deprimidos o las personas que padecen enfermedades crónicas discapacitantes como esclerosis múltiple son ejemplos de poblaciones en riesgo que requieren vigilancia y tratamiento estrechos. Las intervenciones psicosociales como la psicoterapia y la atención en la comunidad, reforzadas por medios tecnológicos, pueden ayudar a reducir los riesgos. La participación de escuelas, ONG, padres de familia y el sistema de salud, con todo el personal prestador de servicios (urgenciólogos, trabajadoras sociales, enfermeras, pacientes entrenados y miembros de la comunidad) es esencial para reducir una endemia de suicidios que acaba con la vida de un millón de personas cada año a nivel mundial, el equivalente a una cada 40 segundos de acuerdo con la Organización Mundial de la Salud. ${ }^{12}$

\section{PREVENCIÓN INDICADA}

Un joven que presenta manifestaciones de aislamiento, pérdida de la motivación o deserción escolar debe ser ayudado con la detección temprana de trastornos mentales; ello ha impulsado la creación de intervenciones comunitarias para incrementar la detección de nuevos casos. Varios países en desarrollo tienen programas bien establecidos de servicios de intervención temprana para las personas con psicosis (SITPP), ${ }^{13}$ que han demostrado que mejoran el pronóstico, reducen los internamientos y la discapacidad de los jóvenes. No sólo es deseable, sino indispensable, contar con ellos.

Estos programas deben permitir un acceso rápido y fácil a los servicios de salud mental, un seguimiento intensivo mediante manejo de casos que favorezcan una adecuada continuidad del tratamiento y la aplicación de tratamientos biológicos ${ }^{14}$ e intervenciones psicosociales basadas en la evidencia.

A la luz de los avances paradigmáticos desde la implementación del concepto de endofenotipo de Gottesman y los ulteriores desarrollos en la neurobiología ${ }^{15-17}$ y ciencias psicosociales aplicadas al estudio de la enfermedad mental, concluiría afirmando que no sólo es posible prevenir la enfermedad mental grave: es urgente e indispensable, un imperativo global de la más elevada prioridad.

\section{REFERENCIAS}

1. American Psychiatric Association. Diagnostic and Statistical Manual of Mental disorders. Fifth edition. Washington DC; 2013.

2. Medina MM, Borges G, Lara C, Benjet C. Prevalencia de trastornos mentales y uso de servicios: resultados de la Encuesta Nacional de Epidemiología Psiquiátrica en México. Salud Ment. 2003; 26: 1-16.

3. Madrigal EA. Fortalecimiento de la Salud Mental en México: recomendaciones para una psiquiatría comunitaria. Salud Mental. 2016; 39: 235-237.

4. Gonzalez S, Gupta J, Villa E, Mallawaarachchi I, Rodriguez M, Ramirez $\mathrm{M}$ et al. Replication of genome-wide association study (GWAS) susceptibility loci in a Latino bipolar disorder cohort. Bipolar Disord. 2016; 18: 520-527.

5. Manjarrez G, Vazquez F, Delgado M, Herrera R, Hernandez J. A functional disturbance in the auditory cortex related to a low serotonergic neurotransmission in women with type 2 diabetes. Neuroendocrinology. 2007; 86: 289-294.

6. Manjarrez G, Cisneros I, Herrera R, Vazquez F, Robles A, Hernandez J. Prenatal impairment of brain serotonergic transmission in infants. J Pediatr. 2005; 147: 592-596.

7. Nicodemus KK, Marenco S, Batten AJ, Vakkalanka R, Egan MF, Straub RE et al. Serious obstetric complications interact with hypoxiaregulated/vascular-expression genes to influence schizophrenia risk. Mol Psychiatry. 2008; 13: 873-877.

8. McGrath JJ, McLaughlin KA, Saha S, Aguilar-Gaxiola S, Al-Hamzawi A, Alonso J et al. The association between childhood adversities and subsequent first onset of psychotic experiences: a cross-national analysis of 23998 respondents from 17 countries. Psychol Med. 2017; 9: 1-16.

9. Araujo-Solís MA, Vázquez-Estupiñán MF. Nutrigenética y nutrigenómica. En: Madrazo JA ed. Nutrición y Gastroenterología Pediátrica. Segunda edición. McGraw Hill; 2016.

10. Niculescu AB, Levey DF, Phalen PL, Le-Niculescu H, Dainton HD, Jain $\mathrm{N}$ et al. Understanding and predicting suicidality using a combined genomic and clinical risk assessment approach. Mol Psychiatry. 2015; 20: 1266-1285.

11. Caspi A, Sugden K, Moffitt TE, Taylor A, Craig IW, Harrington H et al. Influence of life stress on depression: moderation by a polymorphism in the 5-HTT gene. Science. 2003; 301 (5631): 386-389. 
12. Organización Mundial de la Salud. Prevención del suicidio, un imperativo global. Ginebra; 2014.

13. Nolin M, Malla A, Tibbo P, Norman R, Abdel-Baki A. Early intervention for psychosis in Canada: what is the state of affairs? Can J Psychiatry. 2016; 61: 186-194.

14. Lieberman JA, Stroup TS, McEvoy JP, Swartz MS, Rosenheck RA, Perkins DO et al. Clinical Antipsychotic Trials of Intervention Effectiveness (CATIE) Investigators. Effectiveness of antipsychotic drugs in patients with chronic schizophrenia. N Engl J Med. 2005; 353: 1209-1223.
15. The Welcome Trust Case Control Consortium. Genome-wide association study of 14,000 cases of seven common diseases and 3,000 shared controls. Nature. 2007; 447: 661-678.

16. Harrison PJ, Weinberger DR. Schizophrenia genes, gene expression, and neuropathology: on the matter of their convergence. Mol Psychiatry. 2005; 10: 40-68.

17. Gottesman II, Gould TD. The endophenotype concept in psychiatry: etymology and strategic intentions. Am J Psychiatry. 2003; 160 (4): 636-645. 